\title{
El uso de Minas Antipersonal (MAP) por parte de grupos irregulares dentro de las dinámicas del conflicto armado colombiano FARC-EP
}

Fecha de entrega: 09-ago-2019 11:1pa9r. Gẹtb50 de Investigación Francisco de Vitoria

Identificador de la entrega: 1158911809

Nombre del archivo:

20989_Centro_de_Investigacion_Francisco_de_Vitoria_El_uso_de_Minas_Antipersonal_MAP_por_parte_de_grupos_irreg_2028419999.docx (309.23K)

Total de palabras: 6047

Total de caracteres: 34857 


\section{晒 UNIVERSIDAD SANDOTOMAS

\section{(Oficina del Alto Comisionado Para la Paz, 2016)DECIMOQUINTA CONVOCATORIA PARA EL FOMENTO DE LA INVESTIGACIÓN Y LA INNOVACIÓN 2020}

Título del proyecto

El uso de Minas Antipersonal (MAP) por parte de grupos irregulares dentro de las dinámicas del conflicto armado colombiano: FARC-EP1.

\section{Campo de acción}

Transdisciplinariedad - Aporte al PIM

El campo de acción que vincula el proyecto de investigación es: SOCIEDAD,

sector externo, ya que pretende analizar problemáticas regionales, nacionales e El proyecto de investigación relaciona diversas disciplinas de las ciencias sociales internacionales, asuntos de especial interés para la Universidad Santo Tomás, y y vincula a investigadores de la División de Ciencias Jurídicas y Políticas, concretamente para la División de Ciencias Jurídicas y Políticas, en el sentido que adscritos a la facultad de Gobierno y relaciones internacionales y la facultad de se relaciona con Derechos Humanos, ciudadanía, y construcción de política Derecho. De conformidad con las líneas de acción del PIM, es importante destacar pública en y para escenarios de paz. En efecto, se pretende impactar la sociedad a que el proyecto se articula con la línea 3: "proyección social e investigación través del estudio de un problema de connotación humanitaria, con implicaciones pertinente" ya que compromete el factor de responsabilidad social, teniendo en de seguridad en el desarrollo de las comunidades y en el impacto jurídico-político cuenta que mediante la investigación con diversos enfoques disciplinares, se originado en el conflicto interno colombiano, como un arma de carácter estratégico busca impactar un sector sensible de la sociedad, como lo es la comunidad que ha y no convencional, el cual ha impactado y generado consecuencias a la comunidad, resultado afectada por el conflicto armado, concretamente por la utilización de las para lo cual el problema estudiado será abordado de manera ética, crítica y minas antipersonal creativa, siempre con el objetivo de transformar la sociedad.

\section{Articulación con funciones sustantivas y el sector social y productivo}

El proyecto vincula las tres funciones sustantivas, docencia, la responsabilidad social y la investigación y se relaciona con el sector social, ya que desde la investigación se pretende transformar e impactar la realidad y generar cambios positivos sobre la sociedad. De manera integral se propone generar cambios concretos en relación con los compromisos internacionales adquiridos en materia de desminado humanitario, de modo que se aporte de manera eficaz a la construcción de una Paz estable y duradera.

\section{Grupo de investigación}

Línea de investigación en la que se inscribe el proyecto

GEGRI- GRUPO DE ESTUDIOS EN GOBIERNO Y RELACIONES INTERNACIONALES Linea de Seguridad y Justicia Politica

\section{Nombre del Investigador principal}

Nicolás Botero Vásquez

\section{Enlace CvLAC}

http://scienti.colciencias.gov.co:8081/cvlac/visua lizador/generarCurriculoCv.do?cod $\mathrm{rh}=0001101$

$$
\underline{242}
$$

\footnotetext{
${ }^{1}$ FARC-EP. Fuerzas Armadas Revolucionarias de Colombia - Ejército del Pueblo. Organización guerrillera que a partir del año 2016, firmó un acuerdo de paz con el gobierno de Colombia y hoy adopta el mismo nombre como partido político.

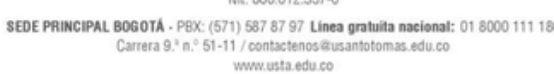

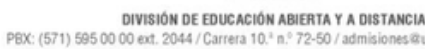

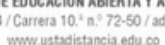

Enlace ORCID

https://orcid.org/0000-0002-2812 $\underline{2814}$
Enlace Google Académico

https://scholar.google.es/citations? view_op=list_works\&hl=es\&user= 1 8FvicAAAAJ 


\begin{tabular}{|c|c|c|c|c|}
\hline \multicolumn{3}{|c|}{ 搯 $\bigcup_{\text {PRIMER CLAUSTRO UNIVERSITARIO DE COLOMBIA }}$} & \multicolumn{2}{|r|}{ Página 2 de 13} \\
\hline División & Facultad & \multicolumn{2}{|c|}{ Programa } & Grupo de investigación \\
\hline Ciencias Jurídicas y Políticas & Gobierno y Relaciones Internacionales & \multicolumn{2}{|c|}{$\begin{array}{l}\text { Gobierno y Relaciones } \\
\text { Internacionales }\end{array}$} & GEGRI \\
\hline Nombre del Co-investigador & Enlace CvLAC & \multicolumn{2}{|c|}{ Enlace ORCID } & Enlace Google Académico \\
\hline Cristhian Fernando Sánchez Giraldo & 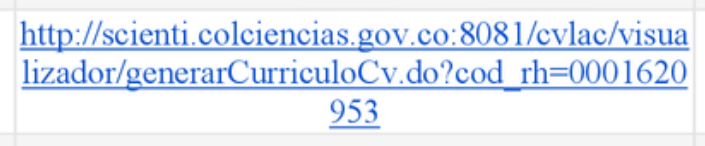 & \multicolumn{2}{|c|}{$\frac{\text { https://orcid.org/0000-0003-2543- }}{\underline{0691}}$} & $\begin{array}{l}\underline{\text { https://scholar.google.es/citations? }} \\
\underline{\text { user=3GUqKOQAAAAJ\&hl=es }}\end{array}$ \\
\hline División & Facultad & \multicolumn{2}{|c|}{ Programa } & Grupo de investigación \\
\hline Ciencias Jurídicas y Políticas & Gobierno y Relaciones Internacionales & \multicolumn{2}{|c|}{$\begin{array}{l}\text { Gobierno y Relaciones } \\
\text { Internacionales }\end{array}$} & GEGRI \\
\hline Nombre del Co-investigador & Enlace CvLAC & \multicolumn{2}{|c|}{ Enlace ORCID } & Enlace Google Académico \\
\hline María Constanza Ballesteros, $\mathrm{PhD}$. & $\frac{\underline{\text { http://scienti.colciencias.gov.co:8081/cvlac/visua }}}{\text { lizador/generarCurriculoCv.do?cod_rh }=0001562}$ & \multicolumn{2}{|c|}{$\frac{\underline{\text { htps: } / / \text { orcid.org/0000-0003-3147- }}}{\underline{7488}}$} & $\begin{array}{l}\text { https://scholar.google.es/citations? } \\
\underline{\text { user=gNTDKPYAAAAJ\&hl=es }}\end{array}$ \\
\hline División & Facultad & \multicolumn{2}{|c|}{ Programa } & Grupo de investigación \\
\hline Ciencias Jurídicas y Políticas & Derecho & \multicolumn{2}{|c|}{ Derecho } & CIFRAVI \\
\hline Nombre del Co-investigador & Enlace CvLAC & \multicolumn{2}{|c|}{ Enlace ORCID } & Enlace Google Académico \\
\hline Pamela Hernández López & $\begin{array}{l}\underline{\text { https://scienti.colciencias.gov.co/cvlac/visualizad }} \\
\text { or/generarCurriculoCv.do?cod_rh }=0001668244\end{array}$ & \multicolumn{2}{|c|}{$\underline{\text { https://orcid.org/0000-0003-1677- }}$} & $\begin{array}{l}\underline{\text { https://scholar.google.es/citations? }} \\
\underline{\text { user=9ez.LN6AAAAA.\&hl=es }}\end{array}$ \\
\hline División & Facultad & \multicolumn{2}{|c|}{ Programa } & Grupo de investigación \\
\hline Ciencias Jurídicas y Políticas & Derecho & \multicolumn{2}{|c|}{ Derecho } & CIFRAVI \\
\hline \multirow{2}{*}{\multicolumn{5}{|c|}{ Resumen de la propuesta }} \\
\hline & & & & \\
\hline \multicolumn{5}{|c|}{ 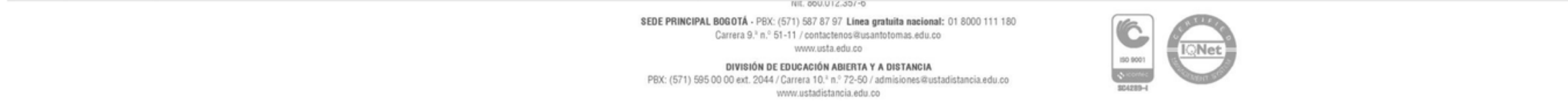 } \\
\hline
\end{tabular}




\section{UNIVERSDDD SANTOTOMAS

Pueblo (FARC-EP), intenta describir y explicar mediante el uso de la teoria de la elección racional, la justificación de carácter estratégico y táctico que utilizó este grupo irregular para el empleo de las MAP o municiones sin explotar, utilizados en las dinámicas del conflicto armado colombiano. La investigación se concentra en el departamento de Antioquia con fuerte presencia de este tipo de armamento no convencional, utilizando las MAP como parte de este grupo irregular. La iniciativa

del proyecto es realizar un análisis académico de la razón por la cual las guerrillas en Minas Antipersonal (MAP), Derecho Internacional Humanitario, desminado Colombia accedieron al uso de este tipo de armamento no convencional dadas lashumanitario, FARC, Justicia Transicional

condiciones asimétricas del conflicto armado e implementaron tácticas inadecuadas

de guerra, violando los protocolos internacionales para la protección a los Derechos Humanos y las infracciones al Derecho Internacional Humanitario, protocolos suscritos por Colombia. Esta será una investigación desligada de valoraciones y juicios politicos, pues no se trata de revivir las atrocidades de la guerra, sino experimentar desde la academia y un enfoque científico, lo adelantado hasta el momento y las metas por alcanzar, según lo acordado por el gobierno de Colombia y el grupo irregular en el punto 4 del "Acuerdo final para la terminación del conflicto y la construcción de una paz estable y duradera", en el que se enmarcan los derechos económicos y sociales para el pleno desarrollo de las comunidades y, así proveerlas de las garantías y condiciones de seguridad necesarias, entre ellas un "programa de desminado"(p.106), el que se inscribe en los procesos de justicia transicional, el cumplimiento y verifícación de los acuerdos internacionales y nacional, además en el compromiso de entrega de armamento de los miembros del grupo armado irregular.

\section{Problema de investigación}

El uso de Minas Antipersonal (MAP) por parte de los grupos irregulares dentro del marco de las dinámicas del conflicto armado colombiano, puntualmente utilizados por las FARC-EP, se presenta como la primera fase de un proyecto de investigación que busca explicar y describir, cuál fue la justificación y uso específico que grupos como las FARC - EP y el ELN, generaron frente a las minas antipersonal dentro de las dinámicas propias del conflicto armado colombiano. También se quiere abordar la problemática actual a la que se enfrenta el Estado colombiano, no solo por la imperiosa necesidad de eliminar y desactivar este tipo de munición que se encuentra en el territorio de difícil acceso, sino, también por los acuerdos internacionales a los que responde Colombia, puntualmente a la Convención de Ottawa. De acuerdo con lo anterior se ha querido plantear la siguiente pregunta de investigación:

¿Cuáles son las características específicas en el empleo de Minas Antipersonal (MAP) por las FARC-EP, en las dinámicas del conflicto armado colombiano?

El problema de investigación de las MAP, en Colombia, es un tema de la agenda nacional e internacional, el que permitirá poner fin a un doloroso capítulo del conflicto colombiano. Adicionalmente, permitirá determinar las responsabilidades de los grupos irregulares que utilizaron estos artefactos, violando los protocolos internacionales y que han dejado a un sinnúmero de personas afectadas de manera permanente y otras fallecidas, según los datos de la Oficina del Alto Comisionado Para la Paz de Colombia. Por último y en relación con esta ofícina del gobierno nacional, el pasado 27 de marzo del año en curso, el país prepara una solicitud de "una nueva prórroga para cumplir con las obligaciones previstas en la Convención de Ottawa sobre la prohibición de minas antipersonal" (Descontamina Colombia, 2019. Párr. 1).
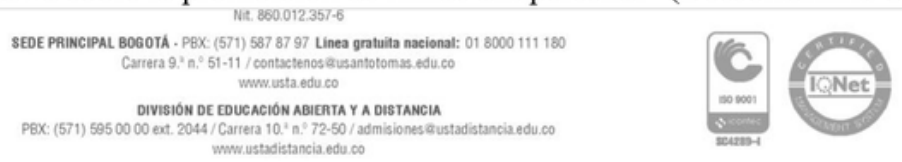


\section{Justificación}

Este proyecto encuentra su razón de ser en el entendido que el proceso de desminado humanitario es clave para la construcción de una paz estable y duradera, toda vez que permite que la población circule libremente sobre los territorios que han sido ocupados por grupos ilegales que para su control han plantado Minas Antipersonal (MAP) artefactos explosivos improvisados (AEI) y municiones sin explotar (MUSE). Esta problemática ha dejado un total de 11.718 víctimas desde 1990 a febrero del presente año, de las cuales $19,5 \%$ han fallecido, de acuerdo con los datos suministrados por la Oficina del Alto Comisionado para la Paz. En razón a lo anterior, es fundamental hacer seguimiento al cumplimiento de los tratados internacionales suscritos por Colombia, tanto en materia de Derecho Internacional Humanitario como en la Justicia Penal Internacional y su aplicación por parte de la Justicia Especial para la Paz en el marco de Justicia Transicional. Con la firma del Acuerdo final para la terminación del conflicto y la construcción de una paz estable y duradera. Las FARC- EP, se comprometieron a la entrega total de las armas y con ello a suministrar al Estado colombiano, la información detallada de ubicación de los artefactos explosivos no convencionales utilizados en las dinámicas del conflicto interno. Es preciso mencionar que el compromiso tiene su origen con el tratado de Ottawa del que Colombia es firmante. En el acuerdo firmado entre las FARC-EP y el gobierno de Colombia, se ha hecho explicito que el grupo irregular colaborará con el fin de cumplir la meta trazada en el proceso de desminado humanitario, inicialmente a 2021. Los grupos armados ilegales, bajo la lógica del combate asimétrico, se organizaron en pequeñas milicias subsanando sus falencias mediante la implementación de tácticas propias de combate, empleando armamento asequible, o, fabricando sus propios dispositivos.

\section{Objetivo general}

Evaluar las características especificas del empleo de Minas Antipersonal (MAP) y los resultados obtenidos en el proceso de desminado humanitario a partir del acuerdo final de paz con las FARC-EP, periodo 2016- 2019.

\section{Objetivos específicos}

- Explicar las razones y justificaciones del empleo de Minas Antipersonal (MAP), en el contexto del conflicto armado colombiano

- Establecer las obligaciones que se desprenden del Acuerdo Final para la Terminación del conflicto y la Construcción de una Paz Estable y Duradera, en relación con el desminado, y los compromisos internacionales adquiridos por Colombia.

- Evaluar el estado actual de una zona en el departamento de Antioquia en proceso de desminado humanitario con el fin de fortalecer la seguridad en el terreno, previo compromiso efectivo en el punto 3 y 4 del Acuerdo Final de Paz entre el gobierno de Colombia y las FARC-EP.

- Explicar la tensión entre penas alternativas y proporcionales en el marco de la justicia transicional para aquellos integrantes que hayan cometido crímenes de guerra y lesa humanidad, específicamente en el uso de las MAP.

Teoría de la Elección Racional.

\section{Estado del arte y marco conceptual}

\section{Estado del Arte}
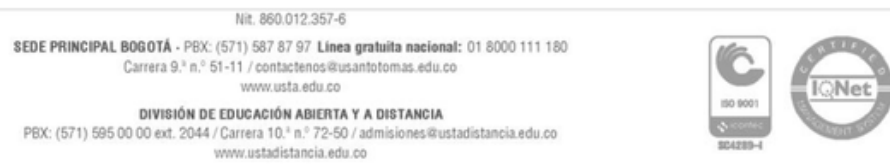


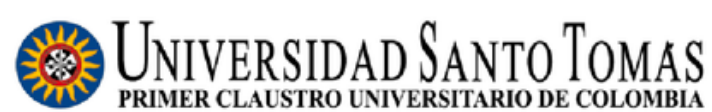

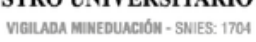

Página 5 de 13

Para poder realizar un análisis riguroso sobre la Teoría de la Elección Racional se revisará su contenido teórico, teniendo en cuenta las diferentes posturas enunciadas por algunos autores, quienes han realizado un asiduo estudio sobre su aplicabilidad o no, en el estudio de las ciencias sociales. Para esto, es necesario dividir este segmento del marco teórico, entre las tesis que están a favor de la Teoría de la Elección Racional y su antítesis, es decir los fundamentos que están en oposición. Posteriormente, se establecerá una síntesis que permitirá determinar si los elementos que sustentan esta teoría realmente pueden llegar a justificar la comprensión y/o explicación de fenómenos sociales. Por último, se presentará un análisis sobre la aplicabilidad de la Teoría de la Elección Racional al objeto de estudio de esta tesis, es decir al emplazamiento de MAP por parte de las FARC, en algunas zonas del territorio colombiano, durante el periodo 2016- 2019.

De ahí que su análisis parta de la importancia de establecer que todos los fenómenos sociales son consecuencia de diferentes interrelaciones que deben desintegrarse para su correcta explicación. Se parte de dar sentido a los fenómenos generales "macro", desde un análisis causal acerca de la interacción entre los elementos que lo integran: fenómenos particulares "micro".

Aplicabilidad de la Teoría de la Elección Racional al emplazamiento de MAP por parte de las FARC, en algunas zonas del territorio colombiano.

Dado que el conflicto interno colombiano, es un fenómeno social que sin lugar a dudas se puede estudiar desde un enfoque analitico, la aplicabilidad de la Teoría de Elección Racional es por supuesto una alternativa que podría coadyuvar a su correcto entendimiento, toda vez que, si bien necesita del respaldo de otras teorías para su sustento, podría encontrar posibles respuestas, en el momento de desglosar tanto las partes que lo componen, como sus posibles relaciones causales. Y es desde este desglose que se permite el cruce de toda clase de variables, partiendo de lo general a lo particular, o viceversa, tal sería el caso de aplicar la violencia directa unilateral de las FARC como variable dependiente, y el uso MAP por parte de esta guerrilla en algunos territorios del país como variable independiente.

Para efectos de esta tesis, la posibilidad de encontrar posibles respuestas a su objeto de estudio, mediante el intento de desglosar el fenómeno social tanto entre las partes que lo componen, como desde un análisis causal acerca de la interacción entre los elementos que lo integran, constituye la aplicabilidad de la TER, dado que todos los fenómenos sociales (fenómenos generales "macro"), son consecuencia de diferentes interrelaciones, las cuales deben ser disgregadas para su correcta explicación. Para ello, se adopta como variable dependiente el uso MAP por parte de las FARC en algunos territorios del país, en donde interactúan variables independientes que se presentan como aquellos factores que explican que se utilice o no en mayor o menor medida las MAP.

Detrás de estos fenómenos particulares, como lo demuestra el uso de MAP por parte de las FARC en algunos territorios del país, se encuentran diferentes acciones humanas individuales, para las cuales Abitbol \& Botero (2005), las describen como decisiones que toman los actores cuando interactúan entre sí, y son estos los miembros del Secretariado de las FARC, y/o los miembros del Estado Mayor Conjunto de las FARC, quienes toman la decisión espacial y temporal sobre su uso, por lo que su juicio se convierte en un mecanismo causal de la acción social, compartiendo las dos propiedades generales que los autores mencionaban: intencionalidad y racionalidad Por lo tanto, en los estudios sobre conflicto y violencia, la TER puede sobreponerse a las fundamentaciones que los conciben como una patología a evitar y, a su vez, de quienes entienden que es un hecho inevitable, afirmación sustentada por Ríos (2016), considerando al Premio Nobel de economía Thomas Schelling (1961), quien determinó que debe ponerse el punto de atención en los elementos causales, es decir en los actores que buscan de manera racional, consciente y motivacional un elemento de justificación en el conflicto, en lo que Rapoport (1960), (citado también por (Ríos, 2016), concebía como comportamiento estratégico, y en el que prima no solo la fuerza real o tangible sino la dimensión potencial, en la que opera la intimidación. Tal y como señala Martínez (2004), las orientaciones de la elección racional, se fundamentan en tres argumentos básicos, ya abordados con anterioridad, los cuales pueden aplicarse al objeto de estudio de la siguiente manera:

1. Los individuos toman decisiones racionales con arreglo a fines, es decir a través de medios destinados a alcanzar un fin racional. Para quienes dentro de las FARC deciden utilizar MAP, tanto la decisión tomada, como los medios destinados, son racionales.
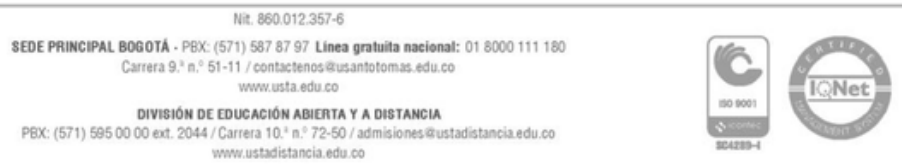


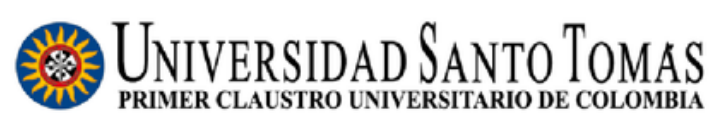 \\ PRIMER CLAUSTRO UNIVERSITARIO DE
VIIILDA MMNEDUACION - SMES: 1704}

Página 6 de 13

2. Dadas sus preferencias (elemento subjetivo de la acción). Quienes dentro de las FARC deciden emplear MAP, a pesar de saber el daño que trae consigo, incluso para sus mismos combatientes, prefieren su utilización dado el "beneficio" que puede representar para la organización en términos estratégicos.

3. Teniendo en cuenta cuáles son las restricciones en las que pueden tomar sus decisiones (elemento objetivo de la acción). Dentro de las restricciones que deberían tenerse en cuenta por parte de quienes toman la decisión dentro de esta organización, de emplazar MAP por diferentes territorios del país, se debe tener en cuenta que pese a la prohibición categórica reglamentada tanto en la legislación nacional, como internacional sobre su uso, este supuesto elemento objetivo de la acción, en lugar de objetarla, dentro de un efecto boomerang, lo que logra es su intensificación, toda vez que este tipo de restricciones no son disuasivas para esta organización.

Por otro lado, y teniendo en cuenta las acciones sociales que Martinez (2004) identifica como una sintesis de la relación existente entre lo objetivo (las restricciones a la acción) y lo subjetivo (preferencias de los actores, incluyendo la interpretación que hagan de la situación), es necesario precisar que en el emplazamiento de MAP, las restricciones dadas por diferentes actores son determinantes a la hora de tomar esta decisión, puesto que pueden incidir en la misma. Entre los que se encuentran no solo a los miembros de las FARC, como lo son los pertenecientes al Secretariado, o al Estado Mayor Conjunto, o cualquier comandante de frente, cabecilla, o guerrillero antiguo o raso, sino también los miembros de la Fuerza Pública tanto oficiales, suboficiales, como soldados y policías, o cualquier miembro del gobierno, y/o los de cualquier rama del poder público del Estado colombiano, así como cualquier integrante de la población civil, entre otros, que hayan intervenido en el proceso de emplazamiento.

Por último, y como el planteamiento de esta tesis supone la interacción de diferentes espacios dentro del territorio colombiano, esta investigación no puede dejar de relacionar la utilización de MAP con la Geografía Política, que supone una congruencia entre el individuo y el ambiente que le rodea, en el caso colombiano donde las características sociales, demográficas, económicas, politicas y culturales son determinantes para que en algunos territorios del pais existan conflictos territoriales. Asimismo, y teniendo en cuenta la tesis que plantean Tollefsen \& Buhaug (2015) (citados en (Ríos, 2016), reivindican con un valor agregado a efectos de entender particularidades locales en su relación con la violencia, la distancia con la ciudad, la disponibilidad de corredores y refugios selváticos o montañosos, así como la distancia socio-cultural con los centros políticos y económicos del país (inaccessibility), se tratará de demostrar que a mayor inaccesibilidad, mayor presencia de miembros del Secretariado de las FARC, mayor presencia de miembros del Estado Mayor Conjunto de las FARC, mayor presencia de estructura de mando de las FARC, mayor presencia de cultivos ilícitos, mayor disputa de rutas con fuente a terceros actores tipo ELN, paramilitares o tipo Fuerza Pública y/o mayor enclaves donde tenían privados de su libertad a secuestrados.

\section{Marco Conceptual}

Las Minas Antipersonal (MAP), han traido consigo un escenario dramático para Colombia, teniendo en cuenta que se encuentran ubicadas en gran parte de su territorio, específicamente en las zonas rurales en donde habitan campesinos, comunidades indigenas y afrodescendientes. Según datos del Alto Comisionado para la Paz, desde 1990 a febrero de 2019, alrededor de 11718 colombianos han muerto o han resultado heridos durante el conflicto armado, (Descontamina Colombia, (27 de marzo de 2019) Colombia se prepara para solicitar nueva prórroga para cumplir con las obligaciones de la Convención sobre la prohibición de minas antipersonal:

Lo anterior a pesar, de que el 6 de septiembre de 2000 Colombia suscribió la Convención de Ottawa, tratado sobre la prohibición del empleo, almacenamiento, producción y transferencia de minas antipersonal y sobre su destrucción. 3 de diciembre de 1997, en donde se comprometió a destruir todas las minas antipersonal que se encuentran en su territorio para el pasado 2011, sin embargo, dada la magnitud del problema, la continuidad del conflicto y la extensión del territorio minado, el Estado Colombiano solicitó una prórroga de 10 años más, lo que implicaba que para el 2021, Colombia debería cumplir las metas previstas. No obstante, recientemente, el gobierno ha comunicado que planea solicitar una nueva prórroga para poder cumplir con el objetivo de retirar y destruir las minas enterradas por los grupos ilegales al margen de la ley, pues a la fecha, solo se ha cumplido el objetivo en relación con las minas plantadas por parte la fuerza pública
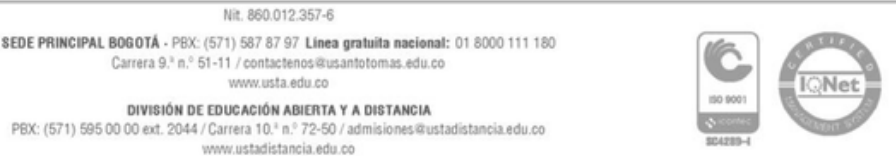


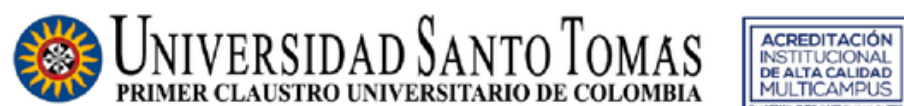

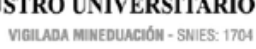

Página 7 de 13

De otro lado, de acuerdo con lo reportado por el Centro Nacional de Memoria Histórica, hasta noviembre de 2016, Colombia ocupaba el segundo lugar, en el mundo, con mayor número de víctimas de Minas Antipersonal (MAP) y Remanentes Explosivos de Guerra (REG), y el primero en el mundo en número de Víctimas pertenecientes a la Fuerza Pública (Centro Nacional de Memoria Histórica, 2017). Sin embargo, tras la firma de los acuerdos de paz con la FARC y el gobierno nacional, Colombia, actualmente ocupa el sexto lugar con víctimas generales y el segundo con víctimas de la fuerza pública.

A pesar que en Colombia está prohibido el uso de las (MAP) Y (REG), pues como ya se ha dicho en apartados anteriores, es uno de los países signatarios del Convenio de Ottawa, es el único país de América Latina en donde los grupos guerrilleros y otros grupos armados ilegales siguen haciendo uso de dichos artefactos como principal estrategia para contener la ofensiva militar y como táctica para evitar la penetración a zonas estratégicas; como lo son cultivos ilícitos y laboratorios (Bejarano, 2010).

Dado que las FARC fue el grupo ilegal que más hizo uso de estas armas no convencionales, según lo registrado en el informe de Monitor de Mina terrestres, es necesario analizar los efectos que ha tenido el acuerdo de paz y su implementación frente al desminado y frente al cumplimiento de los tratados internacionales en la materia.

Dentro del Acuerdo Final, las FARC se comprometió a suministrar información y a colaborar con la tarea de limpieza y descontaminación de los territorios afectados por minas antipersonal (MAP), artefactos explosivos improvisados (AEI) y municiones sin explotar (MUSE). Sin embargo, el compromiso no se agota meramente en proveer información, si se tiene en cuenta que el desminado humanitario está estrechamente relacionado con la erradicación de cultivos ilícitos, la reincorporación a la vida civil de los excombatientes y con la reparación a las víctimas.

En este último punto, es decir, en lo tocante a la reparación a víctimas, es importante mencionar que, a partir del Acuerdo Final para la Terminación del Conflicto, se creó el Sistema Integral de Verdad, Justicia, Reparación y No Repetición. Este sistema parte del principio del reconocimiento de las víctimas como ciudadanos con derechos; derecho a saber la verdad de lo ocurrido, derecho a la justicia; esto es de poder responsabilizar a quienes de manera directa o indirecta se vieron involucrados en graves violaciones del de los derechos humanos y graves infracciones al Derecho Internacional Humanitario; derecho a la reparación y a la no repetición, es decir, el derecho a reparar el daño causado y restaurarse cuando sea posible y garantizar la no repetición de estas vulneraciones.

Es así como frente al tema de responsabilidad de crímenes de guerra y lesa humanidad, como lo es, el empleo de minas antipersonal, existen válidas preocupaciones por parte de las organizaciones internacionales por la imposición de penas alternativas que son comunes dentro del marco de justicia transicional que pueden terminar en impunidad. Vale la pena señalar que el ex secretario de la Naciones Unidas (ONU) Kofi Annan y la Fiscal de la Corte Penal Internacional (CPI) Fatou Bensouda, señalaron en varios medios de comunicación, la necesidad de respetar los estándares internacionales mínimos, así se esté en un marco de postconflicto, pues en caso de impunidad, podrían imponerse sanciones contenidas en el Estatuto de Roma.

Es pertinente anotar que Colombia adoptó dicho estatuto través de la ley 742 del 2002, por medio de la cual se aprueba el Estatuto de Roma y la Corte Penal Internacional, junio 05, DO, 44826. La justicia penal internacional fue creada en el seno de la comunidad internacional para el mantenimiento de la paz, seguridad de los estados y para castigar los crímenes de guerra y lesa humanidad, genocidio y agresión, cuando los Estados no pueden o no quieren actuar, por lo que, su actuación es complementaria.

Bajo este panorama es necesario establecer el estado actual del proceso de desminado humanitario, a partir de los compromisos adquiridos en el acuerdo de paz y sus implicaciones jurídicas en la Justicia Especial para la Paz (JEP), pues este es el órgano competente de juzgar a los autores de los delitos más graves que se cometieron durante el conflicto armado colombiano. Para tal efecto la JEP ha recibido informes por parte de ejército y ex miembros de las FARC de manera que puedan entender el uso de artefactos explosivos no convencionales. Adicionalmente ha escuchado a las víctimas de estas armas ilegales, para comprender el contexto e interpretar los hechos que se van a juzgar.
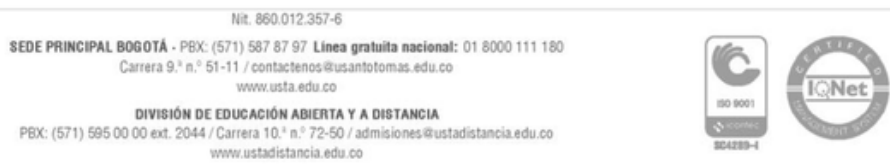


\section{㩊UNIVERSIDAD SANTOTOMAS

\section{Metodología}

El presente proyecto de investigación busca analizar la realidad social y la justificación de carácter estratégico, a la que accedieron los grupos irregulares en Colombia al decidir realizar el emplazamiento de las MAP en determinadas zonas del país y, cuyo resultado es el desminado humanitario que se realiza en el país a 2021. De acuerdo con ello, la investigación se ha fundamentado en el racionalismo crítico a través del análisis y comprensión de los fenómenos sociales y políticos que se presentan en Colombia por el emplazamiento de dichos artefactos. Por esto, se acudirá a un diseño cualitativo, que en palabras de Ispuzua \& Lavia (2016), "puede surgir en diferentes contextos, tanto como primera aproximación (exploratoria, frecuentemente) como directamente ante la definición de una cuestión, aspecto o problema que requiere este tipo de profundización"(p.25), logrando comprender el significado de la realidad de estos fenómenos en términos de la captación de su significado, tales como el método histórico, la entrevista en profundidad y el grupo de discusión, a través del esclarecimiento del significado, del proceso y del contexto, dimensiones estudiadas en este método (Harto de Vera, 2005). Así desde esta lupa no positivista, y poco ortodoxa, -como lo señala Popper (1974) la mayor parte de lo que no puede ser verificado empíricamente si puede ser comprendido y todo lo que puede ser comprendido es significativo (citado en Vera, 2005, p. 82) - se pretende realizar esta investigación.

En el proceso del método histórico, se ha recopilado la información de diferentes fuentes oficiales y de la sociedad civil sobre el emplazamiento de estos tipos de artefactos explosivos y municiones sin explotar, en lo concerniente a las entrevistas se tiene proyectado realizar entrevistas y grupos de discusión con miembros de las Fuerzas Militares y de Policia; miembros de la sociedad civil (que han sufrido este tipo de accidentes), entidades del Estado y desmovilizados, estos últimos que posiblemente hayan estado en las zonas realizando las actividades la implantación de estos dispositivos.

No obstante, a través de la interpretación de las hipótesis que se logren plantear y por el necesario empleo de fuentes y datos a través de la estadística descriptiva y analítica, junto con una descripción cartográfica, se busca reforzar la tesis de investigación desde el enfoque positivista, como lo señala Ríos (2016):

En la interpretación de todas las hipótesis, por el propio uso de fuentes y datos para la investigación, el trabajo termina quedando alineado en un férreo modelo de análisis positivista, que si bien, en ocasiones puede tratarse de una camisa de fuerza interpretativa del objeto de estudio, por otro, en la medida en que se corroboran los resultados planteados, refuerza el alcance y la profundidad de las aseveraciones que se exponen (p. 102).

Conjuntamente, y para realizar un correcto racionamiento en torno al emplazamiento de MAP por casi todo el territorio nacional por parte de las FARC, así como el papel que desempeñó el uso de estas en el Conflicto Armado Colombiano durante el periodo 2002-2015, es necesario realizar un análisis teórico sobre la violencia, fundamentado en las tres tipologías planteadas por Galtung (2003), violencia directa, violencia estructural y violencia cultural, en donde se tratará de adaptarlas al objeto de esta tesis. Racionamiento que tampoco puede dejar afuera una rigurosa exploración de la Teoría de Elección Racional, donde se cristalice la racionalidad de las FARC para utilizar las MAP como instrumentos de lucha en función de las ventajas que les ofreció el territorio nacional.

\section{Resultados esperados}

1. Realizar un diagnóstico sobre la justificación y el uso de las MAP en el conflicto interno colombiano, socializado, a través del Observatorio de Políticas Públicas para el Posconflicto OSEPP (Línea de investigación en seguridad y justicia política).

2. Realizar dos (2) convenios interinstitucionales con:

a. Oficina del Alto Comisionado para la Paz (Colombia)

b. CRI, Centro de Rehabilitación inclusiva de las Fuerzas Militares.
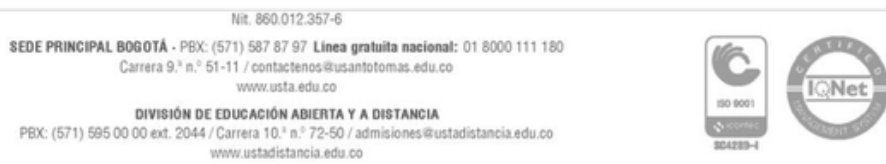


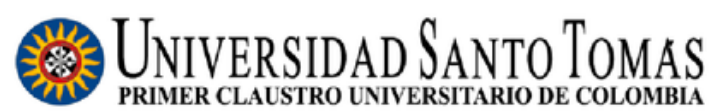

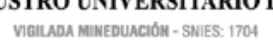

Página 9 de 13

El objetivo de los convenios es socializar los resultados obtenidos en la fase de diagnóstico y al realizar los convenios interinstitucionales con las entidades señaladas, con el fin de que el nuevo conocimiento sea aprovechado en el actual proceso de desminado humanitario en Colombia, como parte del avance entre el sector académico y el gobierno central como un aporte para la construcción de una paz estable y duradera. El primer convenio vincula a la Ofícina del Alto Comisionado para la paz- Descontamina Colombia "dependencia del Departamento Administrativo de la Presidencia de la República" (Oficina del Alto Comisionado para la paz ,2019), como entidad encargada de realizar el seguimiento al proceso de desminado humanitario en Colombia y su trabajo conjunto con otras entidades del Estado, organizaciones de la sociedad civil y las Fuerzas Armadas, relacionadas en el avance del desminado humanitario, esto permitirá a la División de Ciencias Jurídicas y Políticas de la Universidad Santo Tomás, acercarse a la realidad de las problemáticas actuales de las comunidades en las regiones del país, concretamente, en el avance del desminado para la superación de los dilemas del conflicto interno, en zonas periféricas que viven a diario el problema de inseguridad del (terreno) y que quieren superar estas barreras propias del conflicto y la posibilidad de consolidarse en lo económico. Adicionalmente, el segundo convenio, relaciona al Centro de Rehabilitación inclusiva de las Fuerzas Militares (DCRI) (Dirección Centro de Rehabilitación Inclusiva, 2019), que desde sus inicios ha direccionado su estrategia en la rehabilitación integral de los miembros de las Fuerzas Armadas y sus familias. También, es relevante mencionar que su desarrollo e iniciativa se ha consolidado por la decidida ayuda del Estado colombiano, y la cooperación internacional especialmente del Gobierno de Corea producto de la cooperación internacional permanente con ese Estado. El centro de rehabilitación, ha sido uno de los pilares que ha desarrollado uno de los enfoques más interesantes con el fin de rehabilitar, educar y desarrollar diferentes habilidades en los miembros de la Fuerza Pública que han sufrido algún tipo de accidente, apoyando a la reconciliación y el resurgir de una sociedad más inclusiva e incluyente (Dirección Centro de Rehabilitación Inclusiva, 2019). Lo anterior, además de encontrarse presente en los puntos tercero y cuarto del Acuerdo Final para la Terminación del conflicto y la Construcción de una Paz Estable y Duradera, se integra con la actual Política de Defensa y Seguridad (PDS), que busca la articulación de los objetivos estratégicos relacionado en el número 6.1.2. "Proteger a la población y contribuir a su bienestar", coadyuvando a la desarticulación de los grupos ilegales en el país y, el fortalecimiento del desminado humanitario como acción fundamental para el desarrollo de las regiones y de una comunidad en paz y plena convivencia con la ciudadanía en el entorno local y regional (Ministerio de Defensa Nacional, 2019, p.48).

Productos esperados:

1. Realización de un producto de contenido cartográfico sobre el uso de las MAP en el conflicto interno colombiano, el avance de la caracterización geográfica y jurídica política del desminado humanitario en Colombia.

2. Artículo resultado de investigación desarrollado por los integrantes del proyecto para el aprovechamiento del campo objeto de la línea de investigación en seguridad y justicia política, como insumo para el reconocimiento y caracterización en torno al uso de Minas Antipersonal: determinando técnicas de empleo, materiales, cambios y modificaciones originales.

A. Un (1) Artículo resultado de investigación (Q2), sobre el uso y justificación de las MAP en el marco del conflicto interno colombiano y, el proceso de desminado humanitario a 2021.

B. Un (1) Artículo resultado de investigación (Q3) que explique la tensión existente entre las penas alternativas y la justicia transicional y restaurativa derivadas del acuerdo de paz con las FARC-EP.

3. Informe final de investigación a entregar la Unidad de Investigación de la Universidad Santo Tomás.
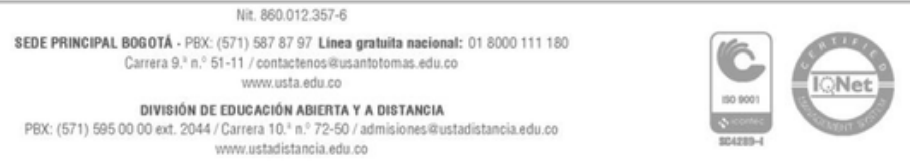


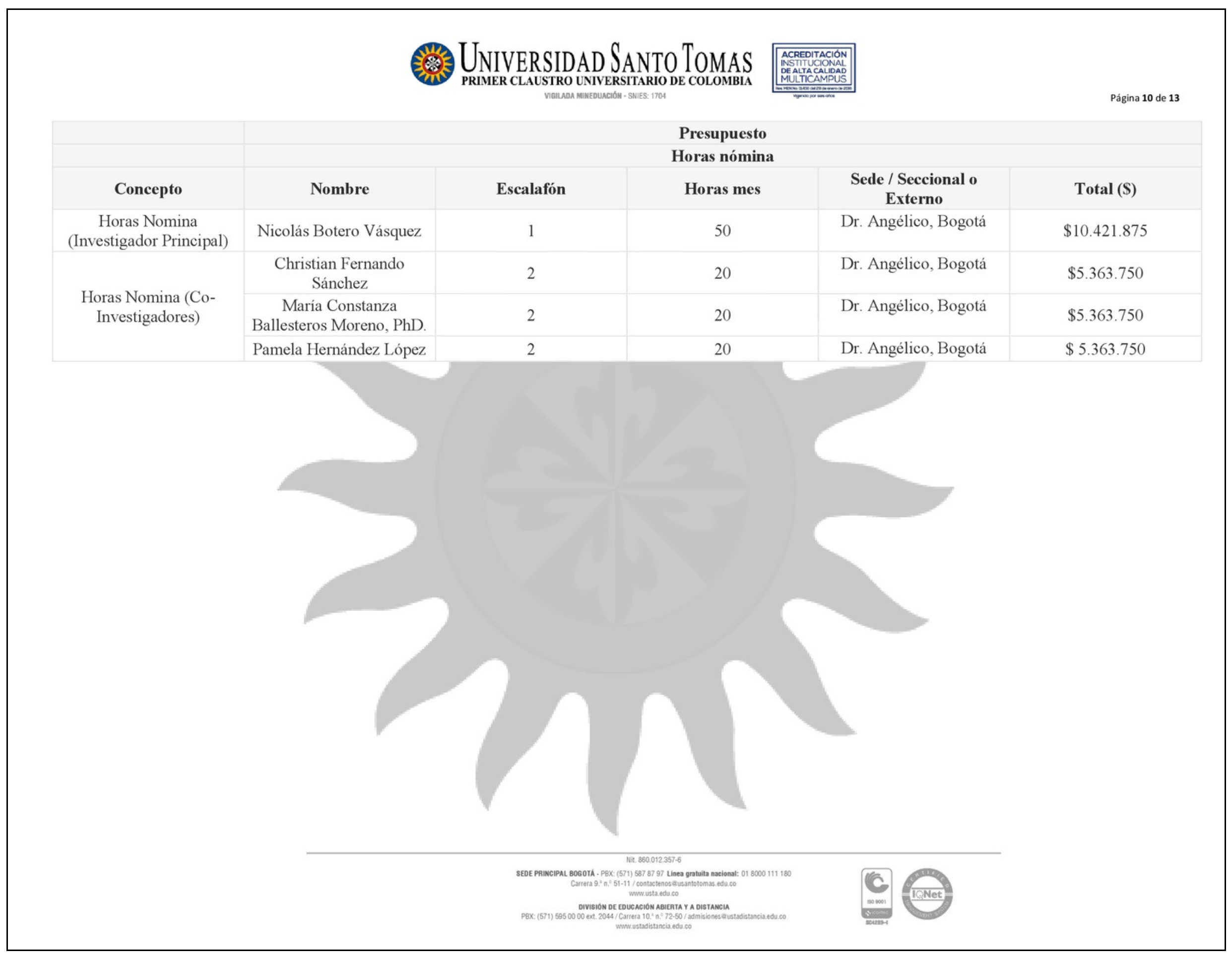




\begin{tabular}{|c|c|c|c|c|c|}
\hline FINANCIACIÓN & RECURSO & VTV PQYEPCI & $\begin{array}{l}\text { Valor } \\
\text { partida }\end{array}$ & $\begin{array}{c}\text { Valor } \\
\text { contrapartida } \\
\text { (Externa) }\end{array}$ & Total (\$) \\
\hline \multirow{4}{*}{ RUBROS } & Servicios Técnicos & WIII PRIMER CLAUSTRO UNIVERSITARIO DE COLOMBIA & 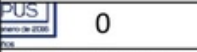 & 0 & $\$ 0$ \\
\hline & Salidas de campo & $\begin{array}{l}\text { Dos visitas al Programa de Prevención e Investigación Contra Minas Antipersonal } \\
\text { PICMA en la ciudad de Medellín. Cada una de estas salidas tendrá la duración de } \\
\text { tres días con el fin de realizar la recolección de evidencia material y científica frente } \\
\text { al empleo de Minas Antipersonal por parte de las FARC-EP, en el departamento de } \\
\text { Antioquia. }\end{array}$ & $\$ 16.011 .380$ & 0,0 & $\$ 16.011 .380$ \\
\hline & Equipos & No se necesitan & 0 & 0 & $\$ 0$ \\
\hline & $\begin{array}{l}\text { Materiales, insumos y } \\
\text { software }\end{array}$ & $\begin{array}{l}\text { Licencias de Atlas TI y Vantage Point, con el fin de realizar y desarrollar desde los } \\
\text { métodos cuantitativos y cualitativos, análisis de resultados estadísticos y técnicos } \\
\text { de la investigación. } \\
\text { Estas licencias deberán instalarse en los computadores institucionales asignados a } \\
\text { los docentes investigadores de la Facultad de Gobierno y Relaciones } \\
\text { Internacionales. } \\
\text { No tiene valor, porque las licencias han sido adquiridas por la Universidad. }\end{array}$ & 0 & 0 & $\$ 0$ \\
\hline \multirow{6}{*}{ BOLSAS } & Papelería & Insumos para las entrevistas, grupos focales y encuestas. & $\$ 1.000 .000$ & 0 & $\$ 1.000 .000$ \\
\hline & Fotocopias & Es posible que se necesite, según el protocolo de investigación. & $\$ 500.000$ & 0 & $\$ 500.000$ \\
\hline & Material bibliográfico & $\begin{array}{l}\text { Es necesario conocer las experiencias del desminado en otros países con conflictos } \\
\text { asimétricos similares. }\end{array}$ & $\$ 2.000 .0000$ & 0 & $\$ 2.000 .0000$ \\
\hline & Auxilio de transporte & $\begin{array}{l}\text { Reuniones y desplazamientos con entidades del Estado nacional y otras oficinas en } \\
\text { la ciudad de Bogotá y Medellin. }\end{array}$ & $\$ 1.104 .154$ & & $\$ 1.104 .154$ \\
\hline & Movilidad & $\begin{array}{l}\text { Es de suma importancia que los investigadores tengan la oportunidad de presentar } \\
\text { los avances de investigación en un congreso asociado a las Ciencias Políticas y } \\
\text { Jurídicas durante el } 2021 \text { como resultado de investigación. } \\
\text { El Congreso Internacional, debe ser indexado con el fin de mejorar el } \\
\text { posicionamiento de los Grupos de Investigación de las facultades FAGORI y } \\
\text { Derecho, por ende, la Universidad Santo Tomás. }\end{array}$ & $\$ 6.118 .200$ & 0 & \\
\hline & $\begin{array}{l}\text { Publicaciones } \\
\text { (Artículos, proceso } \\
\text { editorial y traducción) }\end{array}$ & $\begin{array}{l}\text { - Un (1) Artículo resultado de investigación (Q2), sobre el uso y } \\
\text { justificación de las MAP en el marco del conflicto interno colombiano y, } \\
\text { el proceso de desminado humanitario a } 2021 \text {. } \\
\text { - Un (1) Articulo resultado de investigación (Q3) que explique la tensión } \\
\text { existente entre las penas alternativas y la justicia transicional y } \\
\text { restaurativa derivadas del acuerdo de paz con las FARC-EP }\end{array}$ & $\$ 1.100 .000$ & 0 & \\
\hline \multicolumn{5}{|c|}{ TOTAL DEL PROYECTO: } & $\$ 20.615 .534$ \\
\hline & & 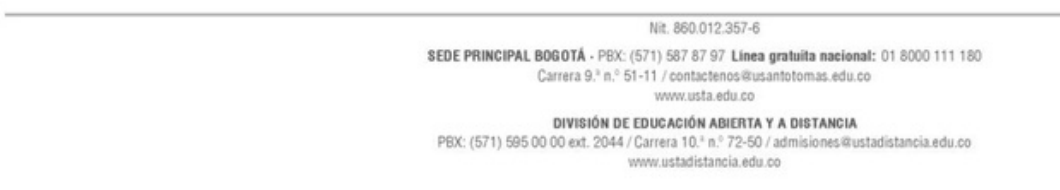 & $(6)$ & & \\
\hline
\end{tabular}




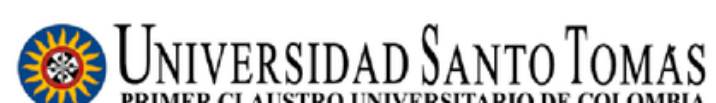 \\ PRIMER CLAUSTRO UNIVERSITARIO DE COLOMBIA \\ VIILLDA MUEEDUACIÓW - SWES: 1704}

\section{Referencia bibliográficas}

Abitbol, P., \& Botero, F. (2005). Teoría de Elección Racional: Estructura conceptual y evolución reciente. Red Colombia internacional, 62, 132-145.

Bejarano Hernández, E. (2010). Minas antipersona, su relación con el conflicto armado y la producción de narcóticos en Colombia. OPERA, 10(10), $263-279$.

Recuperado a partir de https://revistas.uexternado.edu.co/index.php/opera/article/view/3109

Centro Nacional de Memoria Histórica (2017). La Guerra Escondida Minas Antipersonal y Remanente Explosivos en Colombia. Recuperado de

file://C:/Users/coord.espderhumanos/Downloads/la-guerra-escondida\%20(2).pdf

Descontamina Colombia (2019). Oficina del Alto Comisionado para la Paz. Recuperado de http://www.accioncontraminas.gov.co/direccion/Paginas/Quienes-

Somos.aspx

Dirección Centro de Rehabilitación Inclusiva. (29 de 07 de 2019). Dirección Centro de Rehabilitación Inclusiva. Obtenido de Dirección Centro de Rehabilitación

Inclusiva : https://www.dcri.gov.co/index.php?idcategoria=355

Galtung, J. (2003). Violencia cultural. Guernica, España: Gogoratuz.

Harto de Vera, F. (2005). Ciencia Politica y Teoria Politica Contemporánea: Una Relación Problemática Retrieved from

http://data.theeuropeanlibrary.org/BibliographicResource/2000066893671

Hempel, C. (1986). La explicación cientifica. Estudios sobre la filosofia de la ciencia. Barcelona: Paidós.

ISPIZUA, M. \&. (2016). LA INVESTIGACIÓN COMO PROCESO Planificación y Desarrollo. País Vasco , España: Dextra Editorial S.L. doi:ISBN:978-84-16277-67-4

Minsitrio de Defensa Nacional. (Enero de 2019). Política de Defensa y Seguridad- Para la Legalidad, el Emprendimiento y la Equidad. Política de Defensa y Seguridad PDS, 48. Bogotá D.C., Bogotá D.C., Colombia: Ministerio de Defensa Nacional. Recuperado el 05 de 08 de 2019, de

https://www.mindefensa.gov.co/irj/go/km/docs/Mindefensa/Documentos/descargas/Prensa/Documentos/politica_defensa_deguridad2019.pdf
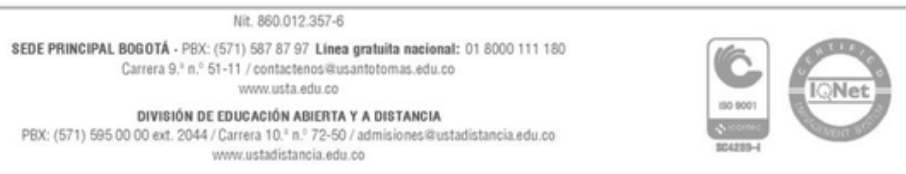


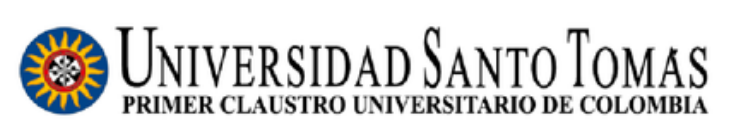

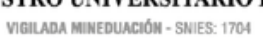

Página 13 de 13

Oficina del Alto Comisionado Para la Paz. (2016). ACUERDO FINAL PARA LA TERMINACIÓN DEL CONFLICTO Y LA CONSTRUCCIÓN DE UNA PAZ ESTABLE Y DURADERA.

Acuerdo Final para la solución del conflicto interno Colombiano, Alto Comisionado para la Paz, Bogotá, D.C. Recuperado el 01 de 08 de 2019 , de

www.altocomisionadoparalapaz.gov.co/

Oficina del Alto Comisionado para la paz. (20 de 07 de 2019). Descontamina Colombia. Obtenido de Descontamina Colombia :

http://www.accioncontraminas.gov.co/prensa/2019/Paginas/190327-Colombia-se-prepara-para-solicitar-nueva-prorroga-para-cumplir-obligaciones-de-

Convencion-de-Ottawa.aspx

Popper, K. (1974). Conocimiento objetivo. Madrid: Tecnos.

República de Colombia y Fuerzas Armada Revolucionarios FARC. (2016). Acuerdo Final para la Terminación del conflicto y la Construcción de un Paz Estable y

Duradera. Recuperado de http://www.altocomisionadoparalapaz.gov.co/procesos-y-conversaciones/Documentos\%20compartidos/24-11-

2016NuevoAcuerdoFinal.pdf

Ríos, J. (2016). Los enclaves de la violencia en Colombia, 1998-2012. Universidad Complutense de Madrid). Retrieved from

http://eprints.sim.ucm.es/37204/1/T37143.pdf

Tollefsen, A. F., \& Buhaug, H. (2015). Insurgency and inaccessibility. International Studies Review, 17(1), 6-25. 10.1111/misr. 12202 Retrieved from

http://onlinelibrary.wiley.com/doi/10.1111/misr.12202/abstract

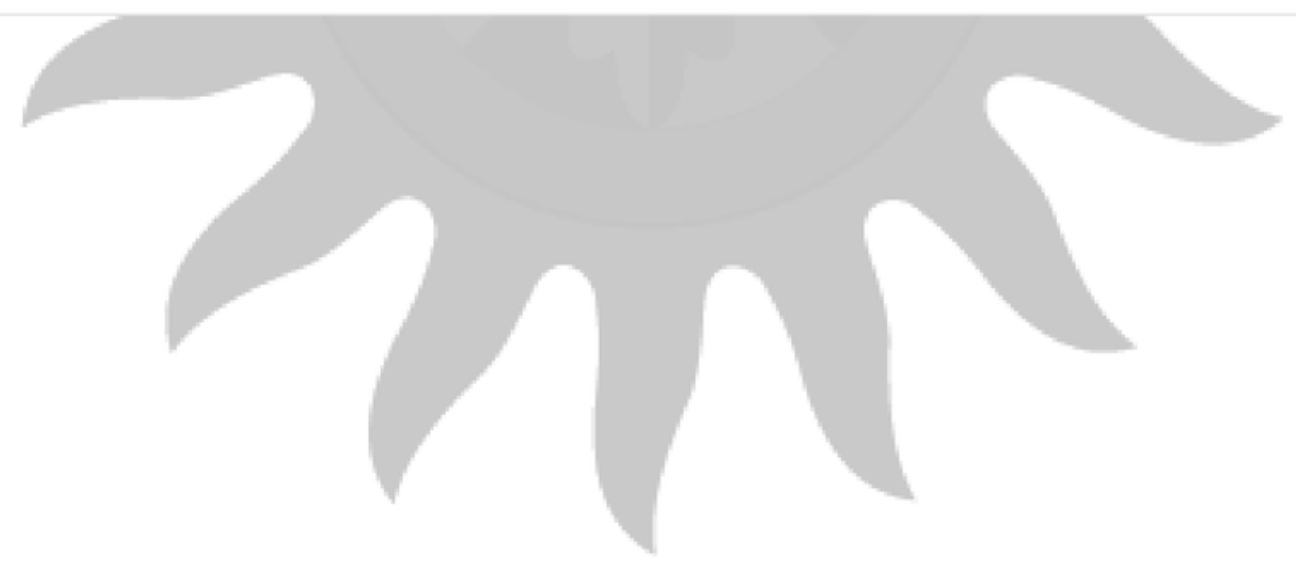

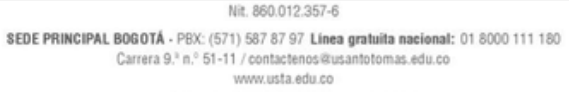

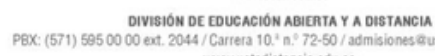

Pext

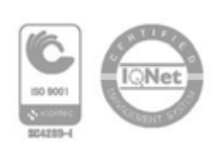


El uso de Minas Antipersonal (MAP) por parte de grupos irregulares dentro de las dinámicas del conflicto armado colombiano FARC-EP

INFORME DE ORIGINALIDAD

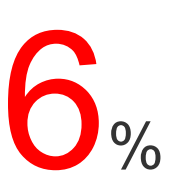

INDICE DE SIMILITUD

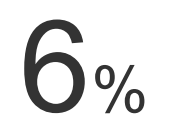

FUENTES DE INTERNET

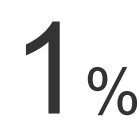

PUBLICACIONES
$0 \%$

TRABAJOS DEL ESTUDIANTE

ENCONTRAR COINCIDENCIAS CON TODAS LAS FUENTES (SOLO SE IMPRIMIRÁ LA FUENTE SELECCIONADA)

$2 \%$

$\star$ www.dejusticia.org

Fuente de Internet

\section{Excluir citas}

Excluir bibliografía 
El uso de Minas Antipersonal (MAP) por parte de grupos irregulares dentro de las dinámicas del conflicto armado colombiano FARC-EP

\section{Instructor}

\section{PÁGINA 1}

\section{PÁGINA 2}

PÁGINA 3 
